\title{
Planet formation in highly inclined binaries
}

\author{
F. Marzari ${ }^{1}$, P. Thébault ${ }^{2}$, and H. Scholl ${ }^{3}$ \\ 1 Dipartimento di Fisica, University of Padova, via Marzolo 8, 35131 Padova, Italy \\ e-mail: marzari@pd.infn.it \\ 2 LESIA, Observatoire de Paris, Section de Meudon, 92195 Meudon Principal Cedex, France \\ e-mail: philippe.thebault@obspm.fr \\ ${ }^{3}$ Laboratoire Cassiopée, Université de Nice Sophia Antipolis, CNRS, Observatoire de la Côte d'Azur, BP 4229, \\ 06304 Nice Cedex, France \\ e-mail: Hans.Scholl@oca.eu
}

Received 23 April 2009 / Accepted 20 July 2009

\section{ABSTRACT}

\begin{abstract}
Aims. We explore planet formation in binary systems around the central star where the protoplanetary disk plane is highly inclined with respect to the companion star orbit. This might be the most frequent scenario for binary separations larger than $40 \mathrm{AU}$. We focus on planetesimal accretion and compute average impact velocities in the habitable region and up to 6 AU from the primary.

Methods. Planetesimal trajectories are computed within the frame of the restricted 3-body problem determined by the central star, the companion star and massless planetesimals. Relative velocities are computed and interpreted in terms of accreting or eroding impacts. Results. We first show that, for binary inclinations higher than 10 degrees, planetesimals evolve, to a first approximation, in a gas-free environment. Planetesimal accretion is confined around the central star in a region determined by two main parameters, firstly by the mutual inclination between the binary plane and the disk, and, secondly, by the binary eccentricity.

Conclusions. The onset of large mutual inclinations between planetesimals due to the nodal randomization causes an increase in the relative velocity. The chances for a successful planet accumulation process depend on the balance between the timescale for node randomization and that of planetesimal accretion. When the binary semimajor axis is larger than 70 AU, planet formation appears possible even for eccentric binaries (up to 0.4). For lower binary separations the region where planetesimals accumulate into protoplanets shrinks consistently. When the mutual inclination between the binary plane and that of the planetesimal disk is larger than $40^{\circ}$, the Kozai mechanism strongly inhibits planetesimal accumulation.
\end{abstract}

Key words. planetary systems: formation - methods: numerical

\section{Introduction}

Planetary formation in binary systems is a complex issue, since each step of the process can be affected in different ways by the companion perturbations. Recent numerical studies (Marzari \& Scholl 2000; Thébault et al. 2004, 2006, 2008, 2009; Paardekooper et al. 2008; Xie \& Zhou 2008) have shown that one stage is particularly sensitive to the presence of the secondary star: the initial accretion of kilometer-sized planetesimals (a review on this topic is given in Haghighipour 2009). Indeed, the coupled effect of secular perturbations of the companion star and friction due to gas in the nebulae induces a size-dependent phasing of orbits which may lead to high impact velocities. This could slow down or even halt the accretion process even in the terrestrial planet region for a wide range of binary separations, i.e., up to $a_{\mathrm{b}} \sim 50 \mathrm{AU}$ for high eccentricity systems (see for example Figs. 8 and 9 in Thébault et al. 2006).

However, these studies are based on the assumption that the planetesimal disk is coplanar to the stellar orbit. Even if this assumption might intuitively seems reasonable, a systematic study by Hale (1994) of binary systems with solar-type components suggests that the spin of the two stars is aligned only for binary systems of 30-40 AU or less. Beyond this distance, the primary's equator, and thus a putative planetesimal disk, appears to be randomly inclined with respect to the binary planet. As a consequence, the inclination between the binary's orbital plane and the circumprimary disk is a parameter that has to be taken into account when studying planetesimal accretion, at least for systems with $a_{\mathrm{b}}>30-40$ AU.

We will focus in this paper on binaries with intermediate separations, i.e. in the 40-100 AU range, exploring the inclination as a free parameter. Similarly to the studies for the coplanar case, the main outcome we are interested in is the impact velocity distribution within the planetesimal population, since this parameter controls the fate of planetesimal collisions, either accretion or erosion. For a significant mutual inclination between the binary orbital plane and the disk of planetesimals embedded in the gaseous disk, the forced inclination due to the companion star might be much more effective in increasing the relative velocities and halting planet formation. As the planetesimals decouple from the gaseous disk and evolve gravitationally, they would feel the binary perturbation and move into inclined orbits. The perturbations of the companion star leads to a progressive randomization of planetesimal node longitudes, starting from the outer region of the disk where the secular periods are shorter. The planetesimal disk gradually evolves into a cloud with an angular opening equal to twice the mutual initial inclination of the disk with respect to the binary plane. We explore in this paper the effects of the nodal randomization on the mutual relative velocities within the planetesimal swarm and on the accretion process. We also estimate the minimum inclination below which planet formation may occur in spite of the binary inclination. 
The paper is organized as follows: in Sect. 2 we show that the planetesimal dynamics perturbed by the companion star keeps the swarm out of the gaseous disk for most of the orbital period. This makes gas drag a negligible perturbation. In Sect. 3 we describe the numerical model used to compute the planetesimal relative velocities. Section 4 is devoted to the analysis of the impact velocities for different binary parameters. In Sect. 5 we derive limiting inclinations for accretion at different binary separations. Finally, in Sect. 6 we summarize our results.

\section{Decoupling between gaseous disk and planetesimals}

Most recent studies of planetesimal accretion in a binary environment (Marzari \& Scholl 2000; Thébault et al. 2006, 2008, 2009; Paardekooper et al. 2008) have focused on the influence of the gaseous component of the disk on particle dynamics. However, the implicit assumption that the planetesimal swarm is embedded in the gas disk is only valid if the disk is coplanar to the binary orbital plane. In this case, planetesimals feel a steady gas drag and have their orbital evolution significantly affected by frictional forces. However, if the companion star is on an inclined orbit with respect to the disk mid-plane, the situation is dynamically more complicated. Three possible scenarios can be envisaged for the interactions between gas and planetesimals:

- Planetesimals form within the gas disk which remains a long-lived coherent entity in spite of the binary perturbations. Numerical simulations with constant viscosity and a polytropic equation of state performed by Larwood et al. (1996) with an SPH code suggest that a disk perturbed by an inclined companion star maintains a coherent structure if the Mach number is lower than 30. It behaves like a rigid body preceding at a rate $\omega_{\mathrm{p}}$ given by:

$$
\omega_{\mathrm{p}}=-\frac{15 M_{\mathrm{s}} R_{\mathrm{d}}^{3}}{32 M_{\mathrm{p}} D^{3}} \cos \left(i_{\mathrm{m}}\right) \Omega(R)
$$

where $M_{\mathrm{s}}$ and $M_{\mathrm{p}}$ are the masses of the secondary and primary star, respectively, $D$ is the radius of the circular orbit of the binary, $i_{\mathrm{m}}$ is the mutual inclination between the disk and the binary orbit, $\Omega(R)$ is the Keplerian frequency and $R_{\mathrm{d}}$ is the disk radius. This equation is derived under the simplified assumption that the disk has a constant density, but it is in general a good approximation to more general cases. In this scenario, when the planetesimals reach the size $(1-10 \mathrm{~km}$ in diameter) for which they evolve under the dominating gravitational force of the two stars, they leave the disk plane because of the forced component in the inclination. Their orbits move in the binary orbital plane and their nodes circulate at different rates, depending on their semimajor axis. Gas drag is probably not a significant perturbation in this scenario, since it affects planetesimal evolution only in the fraction of time during which they cross the gaseous disk plane. This is clearly illustrated in Fig. 1, where we show the projection of the planetesimal positions with respect to the gaseous disk when the inclination of the binary orbital plane is $i_{\mathrm{m}}=20^{\circ}$ with respect to the initial disk plane. Planetesimals spend most of their time out of disk where the gas density is negligible. According to our simulations, for $i_{\mathrm{m}}=30^{\circ}$ planetesimals spend on average only $9 \%$ of their time within one scale height of the gaseous disk. This fraction increases to $13 \%$ when $i_{\mathrm{m}}=20^{\circ}$ and to $27 \%$ when $i_{\mathrm{m}}=10^{\circ}$. As a consequence, we estimate that for $i_{\mathrm{m}} \geq 20^{\circ}$ gas friction can be, to

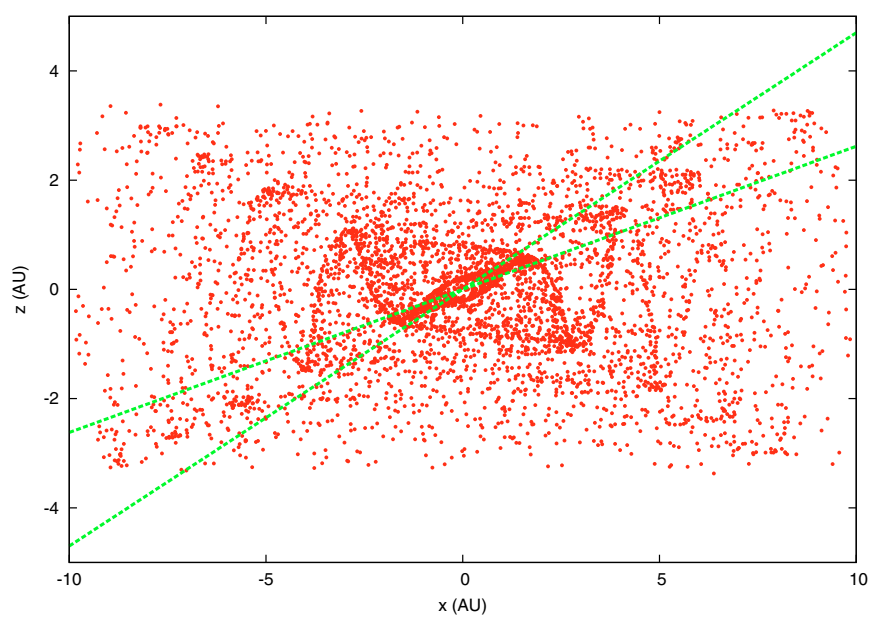

Fig. 1. Planetesimal positions (red squares) in the $x-z$ Cartesian plane after $1 \times 10^{5}$ yrs from the beginning of their gravity dominated evolution. The gaseous disk (assuming a scale height $h=$ $\left.0.05(r / \mathrm{AU})^{(5 / 4)} \mathrm{AU}\right)$ is shown by green dashed lines. The orbital plane of the binary is in the $x-y$ plane and the disk is inclined by $i_{\mathrm{m}}=20^{\circ}$.

a first approximation, completely neglected when computing planetesimal orbital evolution, while the $i_{\mathrm{m}}=10^{\circ}$ case appears as a limiting value below which gas friction has to be taken into account.

- The gaseous disk begins to warp and it is disrupted by binary perturbations. It loses coherence and the gas is dispersed in space. According to Larwood et al. (1996) such disruption by differential precession might affect extremely thin disks. Also in this case, the planetesimals would evolve in a gasfree environment. If the disk is disrupted before the planetesimals detach from the disk then this would be the most significant gas free case, where planet formation would start from a disk of solid material made of small planetesimal precursors which would evolve under gravity only.

- As in the first case, the disk remains coherent but it relaxes to the binary plane on a timescale comparable to the viscous timescale (Larwood et al. 1996). If the process is fast due to a high viscosity of the disk, kilometer-sized planetesimals not have the time to form before the disk relaxes to the binary plane. Planetesimals would then grow when their orbital plane, and that of the disk, are already aligned with that of the binary. In this case any information on the initial inclination would be lost and the system would evolve as a coplanar case (Marzari \& Scholl 2000; Thébault et al. 2006, 2008, 2009; Paardekooper et al. 2008).

Apart from the case of fast relaxation, which possibly occurs in a minority of cases with very high viscosity, in all other cases we expect no or very weak coupling between the gas disk and the planetesimal orbital evolution for binaries with inclination $i \geq 10^{\circ}$. Note that this low- $i$ case with gas drag has been investigated by Xie \& Zhou (2009), who showed that small inclinations between the binary and a circumprimary disc might favor planetesimal accretion as compared to the fully coplanar case.

For our numerical exploration, we will thus make the simplifying assumption that planetesimals evolve in a gas-free environment: the gas-drag force, which introduces a de-phasing of the planetesimal perihelia, does not come into play as in the $2 \mathrm{D}$ case introducing a de-phasing of the planetesimal perihelia. The evolution of the swarm can be described as a pure gravitational $N$-body problem (Thébault et al. 2006) where the relative impact velocity steadily increases because of both the de-phasing 
of perihelia and nodes. In the next section we will numerically compute the evolution of planetesimal relative velocities.

\section{Numerical procedure}

Planetesimal trajectories are computed within the framework of the restricted 3-body problem made up of the central star, the companion star and massless planetesimals. We use the same code as in previous studies of the 2D case (e.g. Thébault et al. 2006, 2008, 2009), since this code is 3D in essence and can handle out of plane perturbers. As already mentioned, the main parameter we are interested in is the evolution of the average impact velocities within the population of test planetesimals. To that effect, our code has a build-in close encounter search algorithm, which tracks at each timestep all 2-body encounters, allowing to precisely compute the relative velocity for each collision (a "collision" being defined as a close encounter within an "inflated radius" equal to $3 \times 10^{-4}$ AU assigned to each particle, see Thébault et al. 2009, for more details). The precision we obtain in our relative velocity estimate is of the order of $5 \mathrm{~m} / \mathrm{s}$ at $2 \mathrm{AU}$.

These values of $\langle\Delta v\rangle$ have then to be interpreted in terms of accreting or eroding impacts. The limit between erosion and accretion is defined by a threshold velocity $v *_{s 1, s 2}$, which depends on the respective sizes $s_{1}$ and $s_{2}$ of the impactors, as well as on the value of $Q *{ }_{s 1, s 2}$, the threshold energy for catastrophic fragmentation. Unfortunately, the parameter $Q *$ is very poorly constrained and estimates found in the literature can differ by up to more than 2 orders of magnitude. We chose here a careful approach and consider that $Q *$ is comprised between 2 limiting values for a "hard" and "weak" prescription. This will in turn result in 2 bracketing values for $v *_{s 1, s 2}$ (see the discussion in Thébault et al. 2006, for more details).

The initial planetesimal swarm is made of 15000 bodies initially set on a 2 -dimensional disk inclined by an angle $i_{0}$ with respect to the binary orbital plane. All the bodies in the disk are started on circular orbits with a semimajor axis ranging from 0.8 to 6.5 AU. All the nodal lines are parallel since all the bodies are clustered in a disk shape. The mass of the primary and secondary stars are fixed to $1 M_{\odot}$ and $0.5 M_{\odot}$ respectively. The binary's orbital parameters $a_{\mathrm{b}}, e_{\mathrm{b}}$ are chosen as free parameters. $a_{\mathrm{b}}$ ranges from 40 to $100 \mathrm{AU}$; beyond those values the perturbations of the companion in the initial phases of planetesimal accretion are too weak. The binary eccentricity $e_{\mathrm{b}}$ assumes different values from 0 to 0.4 . The inclination $i_{0}$ varies from $0^{\circ}$ to $40^{\circ}$. For larger inclinations the Kozai mechanism strongly inhibits planetesimal accumulation, as we will see in the following.

Our initial model setup is based on the assumption that initially the planetesimal swarm forms a flat disk, in other words that it is a dynamically "quiet" system, with all planetesimals $e_{\text {free }}$ and $i_{\text {free }}=0$. For a gas rich environement this choice might be justified by the fact that the progenitors of the km-sized planetesimals are coupled to the gas and cannot have large $e_{\text {free }} 1$. In the present case, this "decoupling" is harder to define, since it could either be the consequence of the planetesimal vertical dispersion around a coherent gas disc (case 1), or the consequence of the gas disc dispersal (case 2). In each case, the relative timing between the arrival of the "initial" kilometre-sized planetesimals, and the decoupling from the gas is difficult to pinpoint. In a worst case scenario, we could have an initial orbital

\footnotetext{
1 Although this issue might be more complex than this simple picture, depending on how planetesimals are formed (see Discussion in Thébault et al. 2006).
}

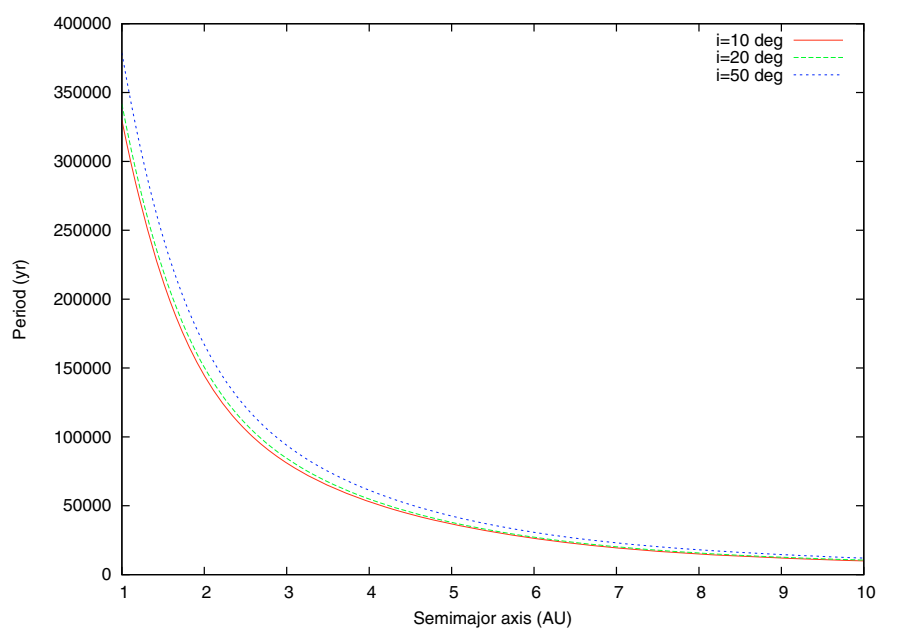

Fig. 2. Circulation period of the nodel longitude as a function of semimajor axis for planetesimals started on a disk around the primary star. The companion star has a semimajor axis $a_{\mathrm{b}}=50 \mathrm{AU}$, and eccentricity $e_{\mathrm{b}}=0$ and different inclinations between the binary orbital plane and the planetesimal disk are considered. The values of the circulation period are computed through direct numerical integration of the equation of motion (3-body problem).

distribution where some planetesimals have an inclination close to $i_{\text {forced }}$ while others are still around $i=0$. This would introduce a high initial free relative velocity component that could not be erased with time (contrary to the gas-rich case, see Fig. 10 of Thébault et al. 2006). This difficult issue clearly exceeds the scope of this paper, but our results should probably be taken as a lower estimate in terms of inhibition of planetesimal accretion.

\section{Results}

\subsection{Dynamical behaviour, encounter velocities}

When the planetesimals feel the binary gravitational pull, their node longitude $\Omega$ starts precessing at a rate which strongly depends on the individual semimajor axis of the bodies as shown in Fig. 2. At the same time, the binary perturbations cause a growth of the eccentricity and a de-phasing of the perihelia. In Fig. 3 we illustrate the orbital distribution of the planetesimal swarm at $t=5 \times 10^{4}$ and $t=10^{5} \mathrm{yr}$ when the binary orbital plane is inclined by $30^{\circ}$ with respect to the planetesimal disk and the eccentricity of the binary orbit is 0.2 (the semimajor axis is $50 \mathrm{AU}$ ). The different timescales of perihelia and node circulation are manifest in the plot. The different degree of randomization of $\varpi$ and $\Omega$ makes it a difficult task to predict the evolution of the relative impact velocity between the planetesimals.

The planetesimal disk moves to the binary orbital plane within one orbital period of the outer planetesimals and gradually loses coherence as a disk. The nodes are randomized and the planetesimal Keplerian orbits take them out of the disk plane. In Fig. 4 we illustrate this behaviour by plotting the positions of the planetesimals at $t=0$, when they are still grouped in a disk, and at $t=1 \times 10^{5} \mathrm{yr}$ when the randomization has disrupted their initial spatial configuration. We adopt this timescale since it is a higher limit for the initial planetesimal accumulation process (e.g. Lissauer 1993)

The randomization of the node longitudes affects the dynamics of the planetesimal population in two ways. The first is that the sparser distribution of the bodies in space leads to a lower impact rate in spite of the growth in the relative velocity. In Fig. 5 


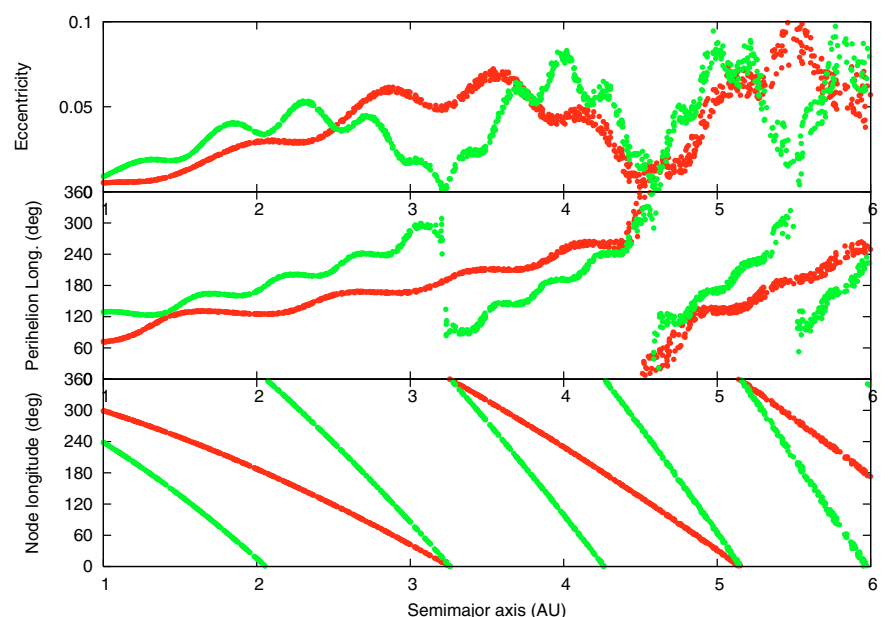

Fig. 3. Distribution of the planetesimal orbital elements at $t=5 \times 10^{4} \mathrm{yr}$ (green dots) and $t=10^{5} \mathrm{yr}$ (red dots). The binary parameters are $i_{\mathrm{b}}=$ $30^{\circ}, e_{\mathrm{b}}=0.2$ and $a_{\mathrm{b}}=50 \mathrm{AU}$.$$
t=10^{5}=0
$$

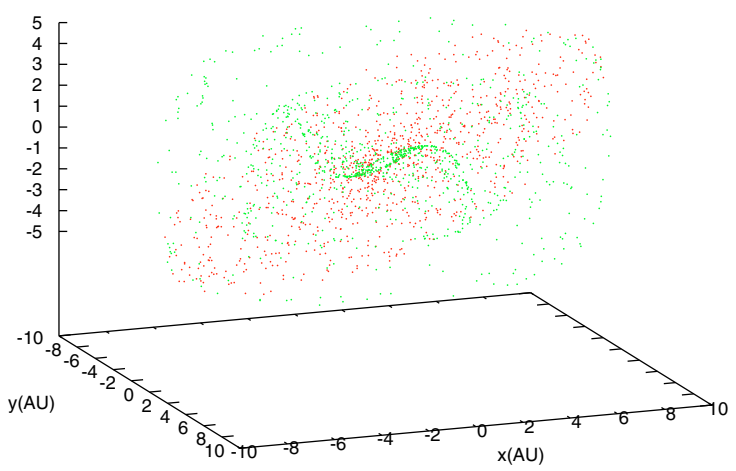

Fig. 4. 3-D spatial distributions of planetesimals at $t=0$ (red dots) and $t=1 \times 10^{5} \mathrm{yr}$ (green dots). The randomization of the nodal lines disrupts the coherence of the initial disk starting from the outer regions. The disk becomes an extended cloud. The binary orbital parameters are $a_{\mathrm{b}}=50 \mathrm{AU}, e_{\mathrm{b}}=0$. and $i_{0}=30^{\circ}$.

we plot the impact rate as a function of time and radial distance. It shows a gradually declining trend as the nodes become more randomly distributed. This trend is more marked at larger distances from the primary star where the nodal randomization is faster. After $10^{5}$ years the impact rate is reduced by $55 \%$ at $1 \mathrm{AU}$ and it drops by $94 \%$ when $r=6 \mathrm{AU}$, independent of the planetesimal size. This percentage can be interpreted as the fractional reduction of the impact rate compared to that of a planetesimal swarm around a single star. Around $t=0$ the impact rate of our model is not yet affected by the binary perturbations and it can be taken as representative of the impact rate around a single star.

The other, and more crucial effect is the progressive increase of impact velocities, as can be clearly seen in Fig. 6. For values of $i_{\mathrm{b}} \leq 40^{\circ}$, this increase is due to the combination of the particle small free eccentricities $e_{\mathrm{fr}}^{2}$ and the large inclination oscillations induced by the inclined companion star. Indeed, the small random horizontal excursion due to $e_{\mathrm{fr}}$ brings in contact bodies having both $i$ and $\Omega$ values increasingly different over

\footnotetext{
2 The existence of a non-zero $e_{\mathrm{fr}}$ component is unavoidable. Indeed, for an unperturbed disc of kilometer-sized planetesimals, equilibrium encounter velocities are of the order of the bodies escape velocities, i.e. a few $\mathrm{m} \mathrm{s}^{-1}$, corresponding to $e_{\mathrm{fr}}$ in the $10^{-5}-10^{-4}$ range.
}

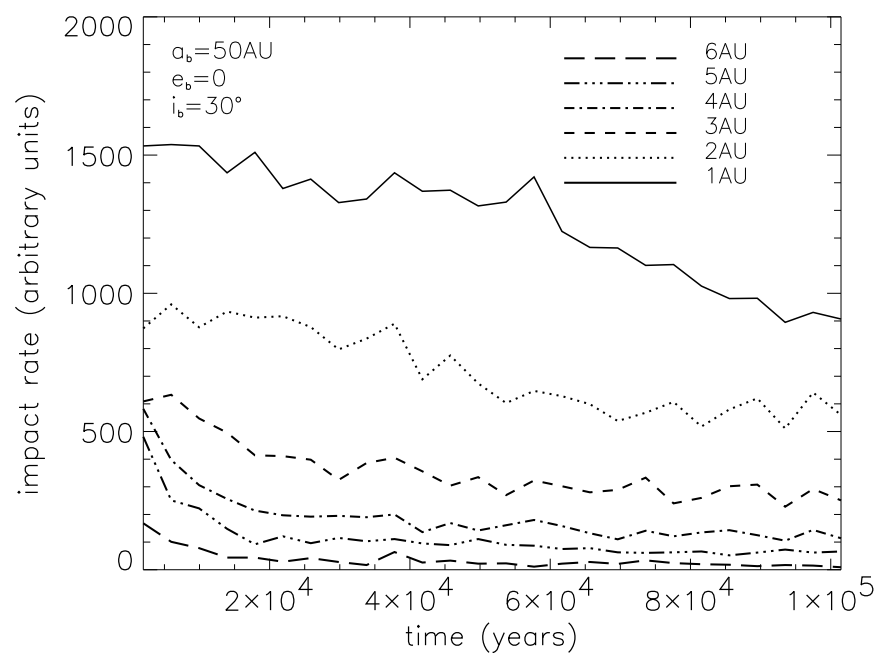

Fig. 5. Average impact rate in the planetesimal swarm as a function of time for the binary configuration: $a_{\mathrm{b}}=50 \mathrm{AU}, e_{\mathrm{b}}=0$ and $i_{\mathrm{b}}=30^{\circ}$. Different curves refer to increasing radial distances from the primary star. The randomization of the node longitudes leads to a sparse planetesimal configuration, leading to a reduction of the encounter rate.

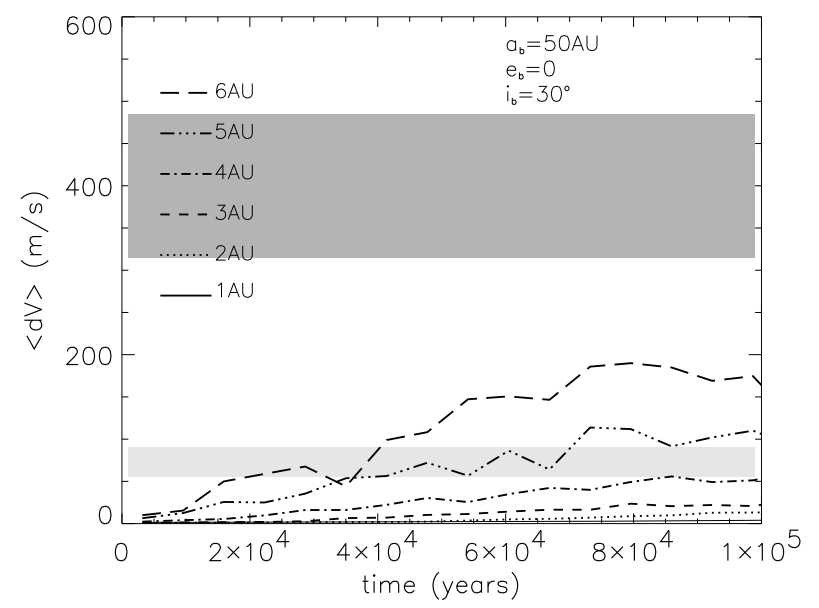

Fig. 6. Average impact rate in the planetesimal swarm as a function of time at different radial distances from the primary star. The grey bands show the erosion limit for planetesimals $1-10 \mathrm{~km}$ (lower band) and $10-50 \mathrm{~km}$ (upper band) in size. The initial inclination between the planetesimal disk and the binary orbit is $i_{\mathrm{b}}=30^{\circ}$.

time as the node oscillations become tighter. This effect is of course more pronounced in the outer regions of the disk, where the pull of the companion star is felt more strongly. Note however that the steady $\langle\Delta v\rangle$ increase is observed everywhere in the $0.8-6 \mathrm{AU}$ region; it is just the pace of this progressive increase that depends on radial distance.

For $i_{\mathrm{b}}>40^{\circ}$ a fully different behaviour is observed and the Kozai oscillations come into play. The eccentricity of the planetesimal orbits begins to grow while the inclination decreases in order to keep the action:

$$
\sqrt{h}=\sqrt{1-e_{\mathrm{pl}}^{2}} \cos \left(I_{\mathrm{pl}}\right)
$$

a constant of motion. The period of oscillation of $e_{\mathrm{pl}}^{2}$ depends on the semimajor axis of the planetesimal orbit and, as a consequence, large differences build up in the distribution of orbital eccentricities of the swarm. This leads to much higher impact velocities as is clearly shown in Fig. 7. 


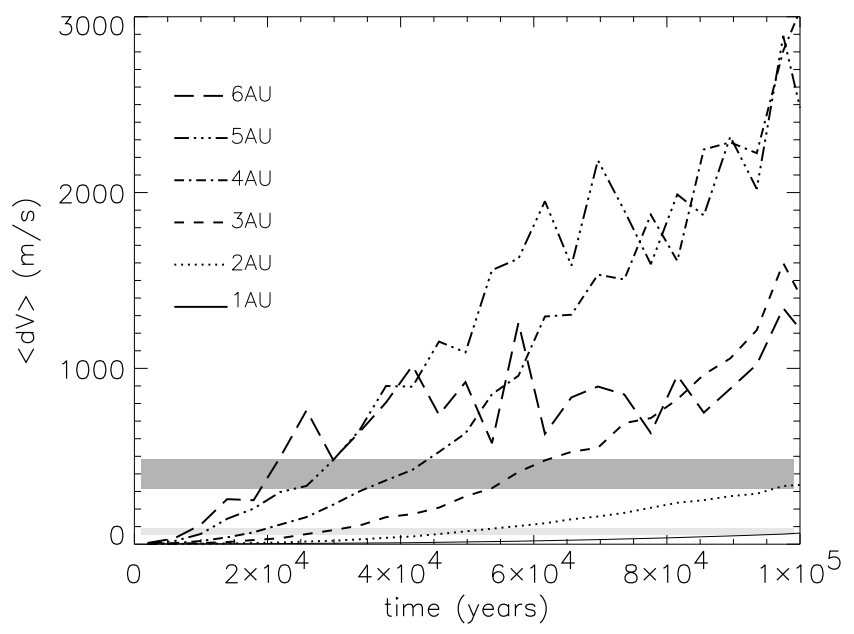

Fig. 7. As in Fig. 6 for an initial inclination of the binary equal to $i_{\mathrm{b}}=40^{\circ}$.

\subsection{Effect on planetesimal accretion}

As illustrated in Fig. 6 and discussed above, the increase of impact velocities is an unavoidable consequence of the node randomization due to the companion star's perturbation that affects all the simulated 0.8 to $6 \mathrm{AU}$ region. However, in spite of this undesired effect, planetesimals might still undergo accretion and form planets. The critical condition is that the timescale for both the mutual velocity growth and impact rate reduction should be long compared to the accretion timescale. More precisely, $\langle\Delta v\rangle$ have to stay at a low, accretion-friendly value long enough so that large planetesimals have enough time to build up. When the high velocity regime is reached, the growing objects have reached a $v *_{s 1, s 2}$ value high enough to have accreting impacts despite higher $\langle\Delta v\rangle$. Of course, studying this effect in detail would require us to follow the evolution of the planetesimal size distribution in addition to their dynamical one. This is unfortunately beyond the reach of deterministic $N$-body codes ${ }^{3}$. We shall thus adopt here a simplified criterion and consider the time at which an averaged $\langle\nu *\rangle$ is reached for two cases: a "small planetesimals" case with $s_{\min }=1 \mathrm{~km}$ and $s_{\max }=10 \mathrm{~km}$ and a "large planetesimals" case with $s_{\min }=10 \mathrm{~km}$ and $s_{\max }=50 \mathrm{~km}$, assuming that planetesimal sizes follow a centered Gaussian distribution between $s_{\min }$ and $s_{\max }$. These two limiting $\langle v *\rangle$ values are indicated by light and dark grey areas in Fig. 6, the width of these areas being due to our careful definition of $v * s 1, s 2$ as being comprised between two extreme values for hard and weak material (see Sect. 3).

As can be seen in Fig. 6 , for our example case with $a_{\mathrm{b}}=$ $50 \mathrm{AU}, e_{\mathrm{b}}=0$ and $i_{\mathrm{b}}=30^{\circ}$, the whole system remains accretionfriendly for a population of large $\geq 10 \mathrm{~km}$ bodies for the duration of the simulation, i.e. $10^{5}$ years, a conservative timescale for runaway growth. For kilometer-sized planetesimals, the situation is less favorable and the $a \geq 5$ AU region becomes hostile to kilometre-sized planetesimal accretion after $\sim 5 \times 10^{4}$ years. In these regions, planet growth can proceed only if in a few $10^{4}$ years planetesimals can grow large enough to have accreting impacts in a $\langle\Delta v\rangle \sim 50-100 \mathrm{~m} \mathrm{~s}^{-1}$ environment. Note however

3 The size evolution of a planetesimal population, under the effects of accreting, cratering and fragmenting impacts, can only be treated in statistical particle-in-a-box codes for which the dynamical modelling is necessarily very simplified. that even if planetesimal accretion is possible, it can probably not lead to the same runaway growth as around an unperturbed single star (Kortenkamp et al. 2001). Indeed, the increase of the impact velocity, even if it cannot stop accretion, significantly slows it down by decreasing the value of the gravitational focusing factor onto growing objects (see the detailed discussion in Thébault et al. 2006). Past this initial planetesimal growth stage, it is difficult to predict the evolution of the swarm at farther stages when large planetesimals and planetary embryos will collide at these high relative velocities. Large relative inclinations might be produced within the planetary system. Quintana et al. (2002) have simulated the formation of terrestrial planets in $\alpha$ Centauri and found that planets may indeed be formed with large mutual inclinations if the orbital plane of the binary is inclined with respect to that of the planetary embryos. However, their simulations start from a coherent and flat disk of protoplanets, while the growing protoplanets might have already developed significant inclinations by the time they collide, according to our scenario. This suggests that the final phase of planet formation in inclined binaries may need additional investigation.

The situation is very different in the $i_{\mathrm{b}}>40^{\circ}$ cases where Kozai oscillations dominate the planetesimal dynamics. As expected, these cases are much more hostile to planetesimal accretion. As can be seen in Fig. 7 (for $a_{\mathrm{b}}=50 \mathrm{AU}, e_{\mathrm{b}}=0$ and $i_{\mathrm{b}}=40^{\circ}$ ), after $10^{5}$ years the impact velocities reach values beyond the erosion limit for both "small" and "large" planetesimals in the whole $a \geq 2$ AU region. Like in the non Kozai case, the rate at which $\langle\Delta v\rangle$ grows strongly depends on the radial distance. As an example, the $\geq 5$ AU region becomes hostile to the accretion of $1-10 \mathrm{~km}$ bodies after only a few $10^{3}$ years, whereas it takes almost $10^{5}$ years for this to be true at $\sim 1 \mathrm{AU}$.

Thébault et al. (2006) derived analytically the timescale before the onset of large impact velocities between planetesimals in planar eccentric binary systems as a function of the binary parameters. In a gas free environment they computed the degree of perihelia randomization required to give high relative velocities and the time needed to reach it. Even if the inclined case may appear similar because accretion occurs in a gas free environment and the relative velocities grow because of the node randomization, it is not possible to derive a similar analytical expression. It is a complex task to estimate how the relative encounter velocity depends on the degree of node dispersion since this is a full 3D problem. In addition, in this scenario both nodes and perihelia, once dispersed, contribute to the grow of the planetesimal relative velocities.

\section{Role of the binary configuration}

In the previous section we have analyzed in detail the dynamical and accretional behaviour of a planetesimal population for two specific test binary configurations. For pedagogical purposes, we chose cases with $e_{\mathrm{b}}=0$ to focus on the effect of the binary inclination and, in particular, on the transition to a Kozai dominated regime when $i_{\mathrm{b}} \geq 40^{\circ}$. We now explore how these results depend on all the binary's orbital parameters $a_{\mathrm{b}}, e_{\mathrm{b}}$ and $i_{\mathrm{b}}$ (the mass ratio between the 2 stars being kept constant and equal to 0.5). For clarity, the accretion/erosion scenario for each binary configuration will be summarized by a single simplified parameter, the semimajor axis $a_{l}$ within which accretion is possible for the $1-10 \mathrm{~km}$ planetesimal population at a threshold timescale of $t_{f} \sim 5 \times 10^{4} \mathrm{yr}$ for the inner zone ranging from 1-3 AU and $t_{f} \sim 1 \times 10^{5} \mathrm{yr}$ for the outer zone extending from 4-6 AU. We assume that planet formation is possible if $\langle\Delta v\rangle$ is lower than $v *_{s 1, s 2}$ for any $t \leq \Delta t_{f}$. Under this condition, larger 
planetesimals can form and resist higher velocity impacts. As an example, by inspecting Fig. 6 we can say that planetesimals beyond $5 \mathrm{AU}$ reach the erosion regime before $1 \times 10^{5} \mathrm{yr}$ while for $4 \mathrm{AU}\langle\Delta v\rangle$ is still below the erosion limit. The inner region within $3 \mathrm{AU}$ always has impact velocities lower than $v *_{s 1, s 2}$ when $t<5 \times 10^{4}$ yr.

The choice of $\Delta t_{f}$ is somewhat difficult and arbitrary. Runaway growth in a planetesimal swarm around a single star is supposed to start after about $10^{4}$ yrs while after $10^{5}$ yrs approximately $33 \%$ of the disk mass is supposed to be in runaway bodies, according to Wetherill \& Stewart (1993). These values cannot be directly applied to the binary case since 1) the binary perturbations increase the relative velocities between the planetesimals, accelerating the erosion rate 2) the collisional frequency decreases with time because of nodal dispersion. In this scenario it is difficult to derive a reliable value for the $\Delta t_{f}$ without knowing the details of the planetesimal accretion process. This would be possible only with a statistical code like the planet building code (Weidenschilling et al. 1997) which, on the other hand, cannot model the perturbations of a companion star. Here we cautiously consider a value of $\Delta t_{f}$ for the inner and outer region of the planetesimal disk which is larger than the runaway growth timescale and should be a good estimate for the time required by planetesimals to grow large enough to sustain further accretion into protoplanets.

The outcome of this analysis is shown in the form of 2-dimensional maps. In Fig. 8 we plot $a_{l}$ vs. $\left(a_{\mathrm{b}}, i_{\mathrm{b}}\right)$ for $e_{\mathrm{b}}=0.0$, $e_{\mathrm{b}}=0.2$ and $e_{\mathrm{b}}=0.4$. The outcome for $i_{\mathrm{b}}=0^{\circ}$ is only a reference value since for low inclinations gas drag comes into play and it must be included in the numerical model (for these low inclination cases, see the recent study by Xie \& Zhou 2009). It is noteworthy that in the analysis of the data it never happens that accretion is possible beyond $4 \mathrm{AU}$ and is prevented within $3 \mathrm{AU}$.

When the companion star is on a circular orbit (Fig. 8a), the effect of inclination is noteworthy only for small values of $a_{\mathrm{b}}$. The secular period of the nodes are short enough to perturb the disk on a timescale comparable to the accretion timescale only for $a_{\mathrm{b}} \leq 50 \mathrm{AU}$. For these small separations and $e_{\mathrm{b}}=0$, planet formation is always possible in the $\leq 3 \mathrm{AU}$ region, except for the Kozai regime at $i_{\mathrm{b}} \geq 40^{\circ}$ Beyond this point the swarm is quickly eroded because of the enhanced relative velocities stirred up by the nodal randomization. At small value of $a_{\mathrm{b}}$ there is a strong dependence of $a_{l}$ on $i_{\mathrm{b}}$, showing that at the origin of the shrinking of the planet formation zone there is the randomization of the nodes. For binary semimajor axes larger than $50 \mathrm{AU}$, the situation is much more favorable to accretion, which can only be stopped, before $t_{f}$, in the Kozai regime with $i_{\mathrm{b}} \geq 40^{\circ}$. This means that $a_{\mathrm{b}} \sim 50 \mathrm{AU}$ is approximately the border value within which secular perturbations alone are efficient enough to affect planet accretion in the non Kozai regime.

For more eccentric binary orbits, the randomization of both nodes and perihelia combine destructively and push the limit for accretion to larger values of $a_{\mathrm{b}}$. As an illustration, Fig. 8c shows the situation for $e_{\mathrm{b}}=0.4$. In the black zone, the relative velocity is beyond the erosion value even at $0.8 \mathrm{AU}$ from the primary star (the inner limit for our planetesimal population). In these cases, the formation of planets, in particular in the outer regions of the disc, is strongly inhibited. For inclinations lower than $\sim 10^{\circ}$, some accretion is possible within 1-2 AU. However, as already stated, our model for $i_{\mathrm{b}} \leq 10^{\circ}$ is less accurate since gas drag may in this case affect the evolution of planetesimals. For $a_{\mathrm{b}}$ larger than $70 \mathrm{AU}$ we retrieve the dependence of $a_{l}$ on $i_{\mathrm{b}}$ and at $a_{\mathrm{b}}=90 \mathrm{AU}$, planetesimal accumulation can only be perturbed in the Kozai regime.
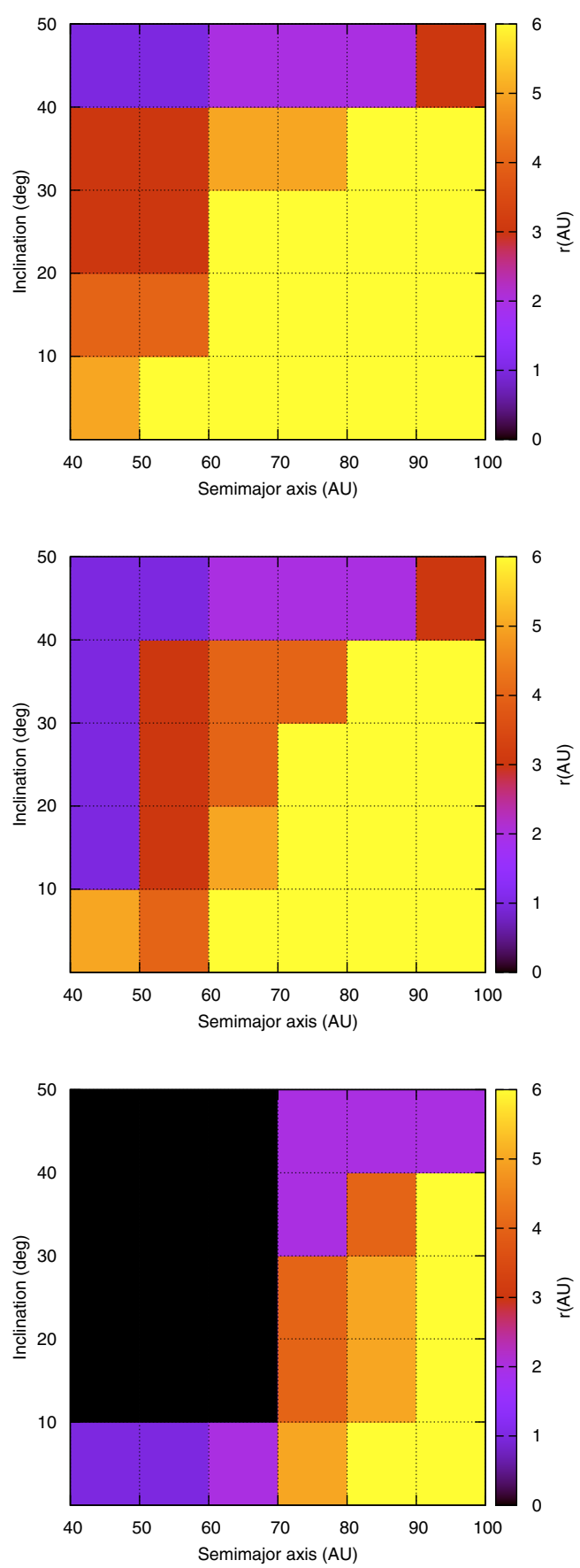

Fig. 8. Maps showing the limiting values for accretion $a_{l}$ as a function of $\left(a_{\mathrm{b}}, i_{\mathrm{b}}\right)$. The top plot refers to the case with $e_{\mathrm{b}}=0.0$, the middle plot to $e_{\mathrm{b}}=0.2$, and the lower plot to $e_{\mathrm{b}}=0.4$. The color coding gives different values of $a_{l}$ (in $\mathrm{AU}$ ), the limiting semimajor axis beyond which planetesimal accretion is possible. Each square of the map refers to the lower value of the labels in the axes. The cases for $i_{\mathrm{b}}=0^{\circ}$ do not include gas drag so they are only indicative.

\section{Conclusions}

In this paper we explore the effect of high $\left(i_{\mathrm{b}} \geq 10^{\circ}\right)$ binary inclination on the planetesimal accretion process. The main outcomes of our work are:

- The gaseous disk and the planetesimals decouple because of the forced inclination of the companion star. As a consequence, planetesimal accumulation should occur in an 
almost gas free environment for inclinations larger than approximately $10^{\circ}$.

- The progressive randomization of the planetesimal node longitudes leads to the dispersion of the planetesimal disk that expands into a cloud of bodies surrounding the star. The sparser configuration leads to a significant reduction in the collisional rate.

- The onset of large mutual inclinations among planetesimals causes an increase of the relative impact velocity that may halt the planet formation process. This effect is particularly strong for $i_{\mathrm{b}} \geq 40^{\circ}$ where the Kozai mechanism comes into play. Below this value, planetesimal accretion might be possible, preferentially in the regions closest to the primary star, depending on the value of $i_{\mathrm{b}}$

- The possibility of planetesimal accumulation depends on the balance between the timescale of node randomization and that of planetesimal accretion. For a binary on a circular orbit, the value of $a_{\mathrm{b}}$ around which this balance occurs is around $50 \mathrm{AU}$. Within this value the secular perturbations are fast enough to induce large relative velocities on a timescale shorter than the typical timescale for planetesimal accumulation. Outside this limit, planetesimals probably have enough time to grow large enough to sustain high velocity impacts.

- When the binary is on an eccentric orbit, the randomization of nodes and periastra can lead to destructive collisions for binary separations up to $70 \mathrm{AU}$.

- The dispersion of planetesimals in the nodal longitude, in those cases where the accretion is effective and lead to protoplanets, possibly leads to planetary systems a) that form on longer timescales because of the reduction of the accretion rate b) on highly mutually inclined orbits.

For binary semimajor axes much larger than those we considered in this paper the nodal longitude randomization becomes much longer. As a consequence, the first stages of planetary formation will probably proceed unaffected by the companion's perturbations: the planetesimal disk is coherent during accumulation into rocky planets and the core of giant planets. In this scenario, planets with a significant inclination with respect to the binary orbit can form. For intial inclinations larger than $\sim 40^{\circ}$, the Kozai cycles may, on a long timescale, lead a planet into a highly eccentric orbit which, at the same time, is very inclined with respect to the equator of the primary. This occurs because during the cycle the inclination with respect to the binary plane is significantly decreased at the eccentricity peak leading the planet far from the equatorial plane of the primary. This might explain the observed orbit of HD 80606b (Wu \& Murray 2003; Gillon 2009; Pont et al. 2009).

Acknowledgements. We thank the referee for useful comments and suggestions that helped to improve the paper.

\section{References}

N. Haghighipour 2009, in Planets in Binary Systems, ed. N. Haghighipour (Springer), to be published

Gillon, M. 2009, MNRAS, submitted [arXiv: 0906 . 4904]

Hale, A. 1994, AJ, 107, 306

Kortenkamp, S. J., Wetherill, G. W., \& Inaba, S. 2001, Science, 293, 1127

Larwood, J. D., Nelson, R. P., Papaloizou, J. C. B., \& Terquem, C. 1996, MNRAS, 282, 597

Lissauer, J. J. 1993, ARA\&A, 31, 129

Marzari, F., \& Scholl, H. 2000, ApJ, 543, 328

Paardekooper, S.-J., Thébault, P., \& Mellema, G. 2008, MNRAS, 386, 973

Pont, F., Hebrard, G., Irwin, J. M., et al. 2009, A\&A, 502, 695

Quintana, E. V., Lissauer, J. J., Chambers, J. E., \& Duncan, M. J. 2002, ApJ, 576,982

Thébault, P., Marzari, F., Scholl, H., Turrini, D., \& Barbieri, M. 2004, A\&A, 427, 1097

Thébault, P., Marzari, F., \& Scholl, H. 2006, Icarus, 183, 193

Thébault, P., Marzari, F., \& Scholl, H. 2008, MNRAS, 388, 1528

Thébault, P., Marzari, F., \& Scholl, H. 2009, MNRAS, 393, L21

Wetherill, G. W., \& Stewart, G. R. 1993, Icarus, 106, 190

Weidenschilling, S. J., Spaute, D., Davis, D. R., Marzari, F., \& Ohtsuki, K. 1997, Icarus, 128, 429

Wu, Y., \& Murray, N. 2003, ApJ, 589, 605

Xie, J.-W., \& Zhou, J.-L. 2008, ApJ, 686, 570

Xie, J.-W., \& Zhou, J.-L. 2009, ApJ, 698, 2066 